\title{
ARTICLE
}

\section{Analysis of Organ Doses from Computed Tomography (CT) Examination by the Radiation Transport Calculation to Develop the Dosimetry System, WAZA-ARI}

\author{
Fumiaki TAKAHASHI ${ }^{1 *}$, Akira ENDO ${ }^{1}$, Kaoru SATO ${ }^{1}$, Takayuki HASEGAWA ${ }^{2}$, Yasushi KATSUNUMA ${ }^{3}$, \\ $\mathrm{Koji}_{\mathrm{ONO}}{ }^{4}$, Takayasu YOSHITAKE ${ }^{5}$, Nobuhiko BAN ${ }^{6}$ and Michiaki KAI ${ }^{6}$ \\ ${ }^{1}$ Japan Atomic Energy Agency, 2-4 Shirakata, Tokai-mura, Naka-gun, Ibaraki-ken, 319-1195, Japan \\ ${ }^{2}$ Tokai University Oiso Hospital, 21-1 Gakkyou, Oiso-mati Naka-gun, Kanagawa-ken, 259-0198 Japan \\ ${ }^{3}$ Tokai University Hospital, 143 Shimokasuya, Isehara-shi, Kanagawa-ken, 259-1193 Japan \\ ${ }^{4}$ Oita Prefecture-Mie Hospital, 3964-1 Miemachi-Miyano, Bungoono-shi, Oita-ken, 879-7101, Japan \\ ${ }^{5}$ Shin-Beppu Hospital, 3898 Tsurumi, Beppu-shi, Oita-ken, 874-0833, Japan \\ ${ }^{6}$ Oita University of Nursing and Health Sciences, 2944-9 Megusuno, Oita-shi, Oita-ken, 870-1201, Japan
}

\begin{abstract}
A new dosimetry system, named WAZA-ARI, is being developed to assess organ doses of a patient in a Computed Tomography (CT) examination. A Japanese adult male voxel phantom (JM phantom) is applied to derive the basic data for dose assessment in WAZA-ARI. The JM phantom defines outer and inner configurations of the human body more precisely than mathematical human models, such as the MIRD-type phantom. The radiation transport in CT examination was simulated with the Particle and Heavy Ion Transport code System, PHITS. A subroutine in PHITS described a source model to simulate emission of photons in a CT machine. This study clarified the relationships between the organ doses and X-ray scanning on a human body with some geometrical conditions.
\end{abstract}

KEYWORDS: CT examination, dosimetry system, WAZA-ARI, JM phantom, organ doses, PHITS

\section{Introduction}

Nowadays, Computed Tomography (CT) is one of the most useful devices in medical imaging techniques. While CT examinations have brought about benefits in diagnosis, this technique delivers significant radiation dose to patients. According to the report by the United Nations Scientific Committee on the Effects of Atomic Radiation (UNSCEAR) in 2000 , CT examinations contributed to $34 \%$ of the collective dose from medical X-rays, although the number of CT examination was only $5 \%{ }^{1)}$ The International Atomic Energy Agency (IAEA) has recently called for enhanced Radiation Protection of Patients (RPoP). ${ }^{2)}$

Dosimetry systems of ImPACT ${ }^{3)}$ and CT-EXPO ${ }^{4)}$ have been applied to avoid unnecessary exposures for patients in CT examinations. The incorporated basic data in the systems were given by radiation transport calculations in the National Radiological Protection Board (NRPB, now the Health Protection Agency) $)^{5)}$ and the National Research Center for Environment and Health (GSF, now the German Research Center for Environmental Health). $\left.{ }^{6}\right)$ Mathematical phantoms (e.g., the MIRD-type phantom ${ }^{7)}$ ) were used in their calculations. ${ }^{8)}$ Techniques and knowledge in numerical analyses have recently progressed for radiation dosimetry. Especially, voxel (volume pixel) phantoms have replaced the mathematical phantoms. ${ }^{9)}$ In addition, new technologies have been introduced for $\mathrm{CT}$ examinations to make accurate diagnosis. From these backgrounds, a new web-based system for clinical practice, named WAZA-ARI, is being developed to enable us to calculate organ doses more accurately than the previous systems and to acquire information about radiation dose to patients from CT examinations in Japan.

*Corresponding Author, E-mail: takahashi.fumiaki@jaea.go.jp (C) Atomic Energy Society of Japan
This paper describes the radiation doses for some geometrical conditions of X-ray source scanning on a human body with the state-of-art dosimetry techniques.

\section{Calculations}

\section{Human models}

The Japan Atomic Energy Agency (JAEA) developed a Japanese adult male voxel phantom of 'JM phantom', which was constructed on the basis of CT images of a healthy volunteer. ${ }^{10)}$ The height and the weight of the JM phantom $(171 \mathrm{~cm}, 65 \mathrm{~kg})$ are nearer to the averaged values over Japanese adult males $(170 \mathrm{~cm}, 64 \mathrm{~kg})^{11)}$ than those of the MIRD-type phantom $(174 \mathrm{~cm}, 71 \mathrm{~kg})$. In addition, the inner configuration of the human body can be precisely defined in the JM phantom, as the voxel size is $0.98 \times 0.98 \times 1 \mathrm{~mm}^{3}$. Figure 1 depicts the large intestine models in the JM and the MIRD-type phantoms. Dose assessments in WAZA-ARI are to be based upon organ doses calculated by the JM phantom.

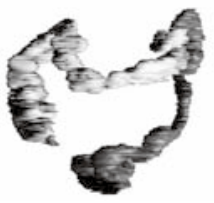

(a) JM-phantom

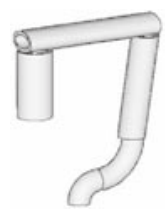

(b) MIRD-type phantom
Fig. 1 Large intestine models in the two phantoms.

The MIRD-type phantom was also applied to the calculations to study an effect of different configurations in the human models on radiation doses averaged over an organ or tissue (hereinafter 'organ doses') in this work. An adult androgynous MIRD-type phantom was constructed by using the software of Body Builder. ${ }^{12)}$ As the original adult phantom by Body Builder was $179 \mathrm{~cm}$ in height, the neck 
model was modified to adjust the height of the phantom in $174 \mathrm{~cm}$ by referring to the model by Yamaguchi. ${ }^{13)}$

The cut off energies of photons and electrons in the radiation transport were set at $1 \mathrm{keV}$ and $10 \mathrm{keV}$, respectively. The PHITS code calculated energies deposited to organs and tissues in the two phantoms. Then, the doses were obtained by dividing the deposited energies by masses of organs and tissues.

\section{Source models in CT examination}

The Particle and Heavy Ion Transport code System

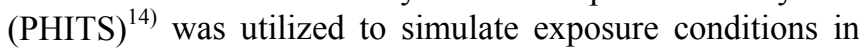
CT examinations. In this work, three source models were defined with a subroutine of 'usrsors' in PHITS. NRPB had previously modeled a source model, in which photons were emitted into a fan-shaped region with a height of $5 \mathrm{~mm}$ from a spot rotating on a circle. ${ }^{5)}$ One of the three source models was referred to this modeling and it is called as a 'one-slice source' in this paper. The one-slice source was shifted every $5 \mathrm{~mm}$ from upper legs to the top of head in the two phantoms. As the height is shorter in the JM phantom than in the MIRD-type phantom, the numbers of calculations with the one-slice sources were 200 and 210 for the JM and the MIRD-type phantoms, respectively.

In addition, two source models were applied to simulate X-ray scanning from chest to abdomen with a range of 48 $\mathrm{cm}$. Both of them are generally termed as 'scanning sources'. Figure 2 depicts images for paths of spots emitting photons of scanning sources in the two types. Here, the horizontal direction corresponds to the vertical axis of the human body (the phantom in the calculation). While the spot moves on circle orbits in the 'non-helical type', the spot moves on a helical curvature in the 'helical type'. Photons were emitted into a fan-shaped region with a height of $5 \mathrm{~mm}$ from the spots in the both types. Several types of scanning were hypothesized to set a pitch of the spot in one circle along the vertical axis of the phantom ('p' in Fig.2) in $5 \mathrm{~mm}, 1 \mathrm{~cm}$ or $2 \mathrm{~cm}$. The start point of the path is set to one of the front, the back, the right or the left of the phantom in the helical type.

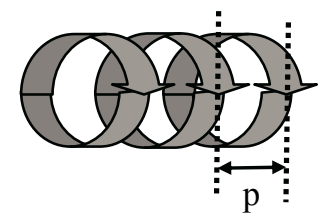

(a) Non-helical

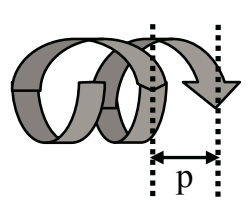

(b) Helical
Fig. 2 Images for path of the spot emitting photons (X-rays) in the two types of scanning sources.

A report of $\mathrm{NRPB}^{15)}$ summarized X-ray tube voltage, focus to axis distance and flat filters for shaping X-ray energy spectrum in some CT machines, which were taken into account for their database of organ doses. Among them, all of the source models in this study referred to the condition in a machine without a bow-tie filter, for which no published data are available. The spot emitting photons rotated around the JM or the MIRD-type phantom on a circle with a radius of $76.0 \mathrm{~cm}$. In addition, a cylindrical region of air with a radius of $5 \mathrm{~mm}$ is set at the rotation center of the source model. The heights of the cylinders are set to $5 \mathrm{~mm}$ and $48 \mathrm{~cm}$ for the one-slice source and the scanning sources, respectively. Two filters of $2.2 \mathrm{~mm}$ thickness aluminum and $0.1 \mathrm{~mm}$ copper were added in the X-ray tube. The software of $\mathrm{xcomp} 5 \mathrm{r}^{16)}$ gave the energy distribution of emitted X-rays accordance with the added filters.

\section{Results and discussions}

\section{Organ doses calculated by the one-slice source}

Figure 3 compares the organ doses for each position of the one-slice sources between the two phantoms. All of the organ doses were normalized to the air kerma at the cylinder region in this paper. The position of the one-slice source is

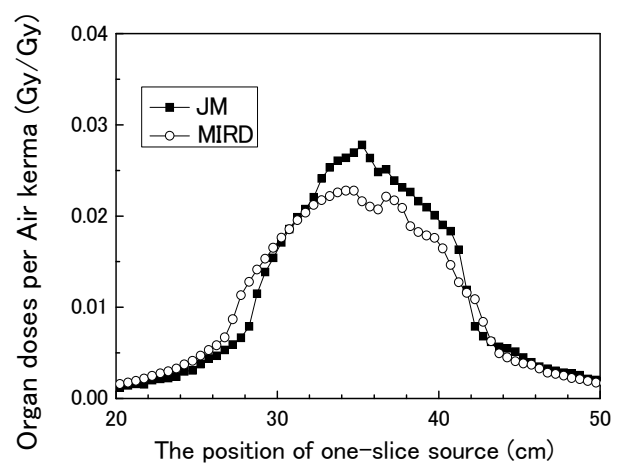

(a) Stomach

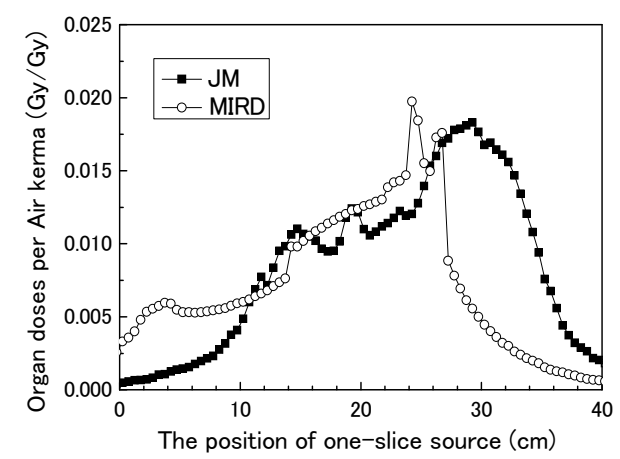

(b) Large intestine

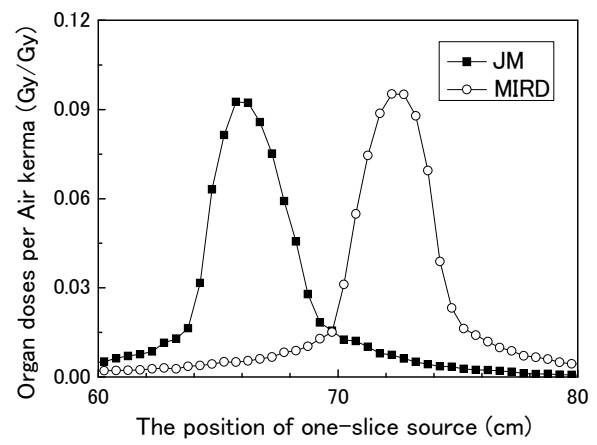

(c) Thyroid

Fig. 3 Relationships organ doses and the position of the one-slice source in the two phantoms. 
indicated with the vertical axis (z-axis) in the MIRD phantom, where $0 \mathrm{~cm}$ is set at the trunk bottom and the upper position has the larger value. The value for the vertical axis of the JM-phantom was set so as to give similar relationships between the dose and the movement of the one-slice source to the MIRD-type phantom about the liver and the stomach.

The dose for the stomach is slightly larger in the JM phantom than in the MIRD-type phantom. The positions with the peaks of the doses are different about the large intestine and the thyroid between the two phantoms, although the maximum values of the doses are almost same. Table 1 lists the position of some organs for the vertical axes in the two phantoms. The range is divided into $5 \mathrm{~mm}$ to be accordance with the position of the one-slice source here.

Table 1 The ranges of organs on the vertical axis

\begin{tabular}{lcc}
\hline Organs & $\mathrm{JM}(\mathrm{cm})$ & $\mathrm{MIRD}(\mathrm{cm})$ \\
\hline Adrenals & $35.0-40.0$ & $38.0-43.0$ \\
Large int. & $8.0-36.5$ & $0.0-27.0$ \\
Liver & $29.0-45.0$ & $27.0-43.0$ \\
Stomach & $28.0-42.5$ & $27.0-43.0$ \\
Thyroid & $64.0-69.0$ & $70.0-75.0$ \\
\hline
\end{tabular}

The organs were directly irradiated to primary photons from the one-slice source only within the fan-beam region. Figure 4 shows a significant dependence of the dose on the mass fraction of the organ in the region of one-slice source at each height for the JM phantom. If only a part of organ was involved in the fan-beam region, the dose increased with the mass involved. The dose of large intestine was, however, different between the upper region around $30 \mathrm{~cm}$ and the lower region around $15 \mathrm{~cm}$, although the mass fractions are

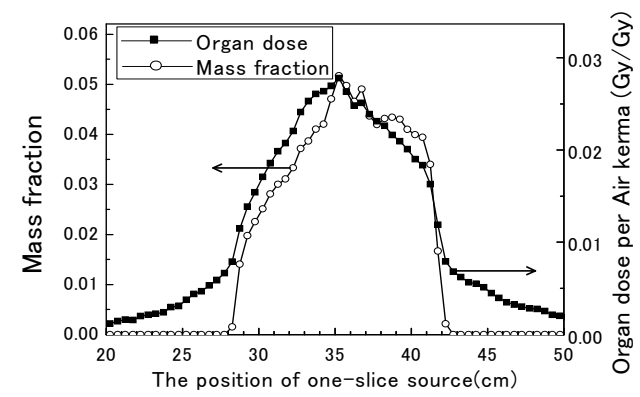

(a) Stomach

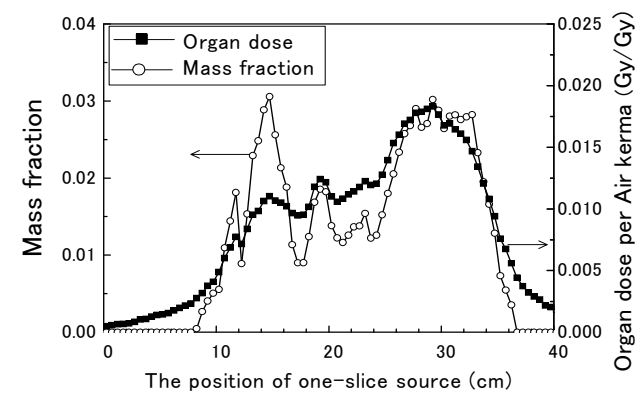

(b) Large intestine

Fig. 4 Doses and mass fraction of organs in the JM phantoms at each height of the one-slice source. almost the same at these positions. This reason relates to the shape of the large intestine, as depicted in Fig.1 (a). While the ascending and descending colons are located near the trunk surface, the sigmoid colon is located around the center of trunk. Thus, the position brought about the different dose.

\section{Organ doses calculated by the scanning source}

Table 2 lists some of the organ doses by the scanning source in the non-helical type with the moving pitch of $5 \mathrm{~mm}$. In this case, the scanning source can describe equivalent condition to accumulate 96 one-slice sources along the vertical axis of the phantom. Thus, the doses in Table 2 agreed well the values, which were obtained by integrating the organ doses by the one-slice sources over the range from $18 \mathrm{~cm}$ to $66 \mathrm{~cm}$ in Fig.s 3 and 4.

Different configurations in the phantoms did not affect the doses to the organs, which were wholly involved within the scanning source range for the both phantoms. For example, the stomach doses by the two phantoms agreed within $7 \%$ each other, although Fig.3 (a) showed a slight difference of the stomach dose between the two phantoms. On the other hand, the large intestine and the thyroid were located also outer of the scanning source region. Especially, the thyroid was not involved in the interested region for the MIRD-type phantom. Thus, the thyroid dose by the JM phantom was about 7 times larger than that by the MIRD-type phantom. The data by the MIRD-type phantom were near to the organ doses utilized in ImPACT. ${ }^{3), 15)}$ The results imply that WAZA-ARI can give different organ doses from ImPACT even for the same scanning range.

Table 2 The organ doses per air kerma for X-ray scanning from

\begin{tabular}{lcc}
\multicolumn{3}{c}{ chest to abdomen in the two phantoms ${ }^{(1)}(\mathrm{Gy} / \mathrm{Gy})$} \\
\hline Organs & $\mathrm{JM}$ & $\mathrm{MIRD}$ \\
\hline Adrenals & $0.534(1.00)^{(2)}$ & $0.577(1.00)$ \\
Large int. & $0.533(0.71)$ & $0.337(0.58)$ \\
Liver & $0.685(1.00)$ & $0.684(1.00)$ \\
Stomach & $0.716(1.00)$ & $0.682(1.00)$ \\
Thyroid & $0.403(0.46)$ & $0.062(0.00)$ \\
\hline
\end{tabular}

(1) Non-helical type, the moving pitch ('p' in Fig. 2(a)); $5 \mathrm{~mm}$

(2) The fraction of mass involved in the source region

Table 3 summarizes some of the organ doses, which were calculated with the JM phantom and the different scanning sources. The start point of the helical type means the position of the spot emitting photons for the phantom at the lowest height of the source. The masses of the stomach and adrenals are about $10 \%$ and $1 \%$ of the liver, respectively. The large intestine, which is located also lower region of the scanning source, has larger mass in the interested region than the stomach.

The path of scanning source affected the incident position of photons on the trunk. The doses of liver, stomach and large intestine, however, were not significantly changed by the definition of the scanning source. On the contrary, the adrenal doses changed about $20 \%$ even for the same moving pitch in one circle, if the start point of the spot emitting photons was set to different positions in the helical type. The 
dose decreased for the condition, where the spot emitting photons was moving around the front side at the height of the adrenals. It is assumed from the result that the path of scanning source can affect also doses to other small organs, such as ovaries in a female.

Table 3 The organ doses per air kerma for X-ray scanning from chest to abdomen in the JM phantoms (Gy/Gy)

\begin{tabular}{lcccc}
\hline Organs and & Start point & \multicolumn{3}{c}{ Organ doses per air kerma } \\
Source type & (Helical type) & $\mathrm{p}=5 \mathrm{~mm}^{(1)}$ & $\mathrm{p}=10 \mathrm{~mm}$ & $\mathrm{p}=20 \mathrm{~mm}$ \\
\hline a) Adrenals & & & & \\
Non-helical & & 0.534 & 0.547 & 0.492 \\
Helical & Right side & 0.462 & 0.513 & 0.523 \\
Helical & Back & 0.570 & 0.495 & 0.488 \\
Helical & Left side & 0.557 & 0.581 & 0.559 \\
Helical & Front & 0.504 & 0.534 & 0.551 \\
\hline b) Large int. & & & & \\
Non-helical & & 0.533 & 0.533 & 0.538 \\
Helical & Right side & 0.525 & 0.529 & 0.534 \\
Helical & Back & 0.536 & 0.524 & 0.529 \\
Helical & Left side & 0.522 & 0.529 & 0.527 \\
Helical & Front & 0.524 & 0.538 & 0.525 \\
\hline c) Liver & & & & \\
Non-helical & & 0.685 & 0.686 & 0.685 \\
Helical & Right side & 0.682 & 0.682 & 0.687 \\
Helical & Back & 0.688 & 0.692 & 0.685 \\
Helical & Left side & 0.681 & 0.689 & 0.682 \\
Helical & Front & 0.682 & 0.688 & 0.682 \\
\hline d) Stomach & & & & \\
Non-helical & & 0.743 & 0.760 & 0.741 \\
Helical & Right side & 0.705 & 0.740 & 0.734 \\
Helical & Back & 0.694 & 0.740 & 0.734 \\
Helical & Left side & 0.729 & 0.699 & 0.719 \\
Helical & Front & 0.717 & 0.712 & 0.731 \\
\hline (1) The & & &
\end{tabular}

(1) The moving pitch ('p' in Fig.2)

\section{Conclusion}

The radiation transport calculations by PHITS clarified the organ doses for some geometrical conditions of CT examinations. The organ doses by the JM phantom can be considered as more appropriate data for a dose assessment of a Japanese male adult. It is assumed from the analysis that WAZA-ARI can give different doses from the other systems, such as ImPACT, even for a same scanning range over a body. More studies are to be made on the relationships between the scanning and the organ doses for many types of machines. The scanning source model can be applied to derive the organ doses, according to the characteristics of any CT machines. Thus, it is be expected that a dose assessment method can be established for CT examinations in WAZA-ARI based upon the analysis using the source model. Moreover, the attenuation of photons in a bow-tie filter will be taken into account for the source model.

\section{Acknowledgment}

This work has been supported by the Ministry of
Education, Science, Sports and Culture (MEXT), Grant-in-Aid for Scientific Research (C), 20591484, 2008.

\section{References}

1) UNSCEAR, United Nations Scientific Committee on the Effects of Atomic Radiation 2000 Report to general assembly -Annex D: Medical radiation exposures-, United Nations (UN) (2000).

2) IAEA, http://rpop.iaea.org

3) Imaging Performance Assessment of CT scanners (ImPACT), http://www.impactscan.org/ctdosimetry.htm

4) D. Stamm, H. Negel, "CT-EXPO, ein neuartiges Programm zur Dosisevauluierung in der CT,“ Fortshr. Roentgenstr., 174, 1570 (2002), [in German].

5) P. C. Shrimpton, D. G. Jones, M. C. Hillier, et al., Survey of CT practice in UL -Part II: Dosimetric aspects-, NRPB-R249, National Radiological Protection Board (NRPB) (1991).

6) M. Zankl, W. Panzer, G. Drexler, The calculation of dose from external photon exposure using reference human phantoms and Monte Carlo methods -Part VI: Organ doses from Computed Tomographic examinations-, GSF-Bericht 30/91, GSFForschungzentrum (GSF) (1993).

7) M. Cristy, Mathematical phantom representing children of various ages for use in estimates of internal doses, NUREG/CR-1159, Oak Ridge National Laboratory (ORNL) (1980).

8) ICRU, Patient dosimetry for $X$ rays used in medical imaging, ICRU Report 74, International Commission on Radiological Units and Measurements (ICRU) (2005).

9) H. Zaidi, X. G. Xu, "Computational anthropomorphic models of the human anatomy: The path to realistic Monte Carlo modeling in radiological sciences," Ann Rev., Biom, Engin., 9, 471 (2007).

10) K. Sato, H. Noguchi, Y. Emoto, et al., "Japanese adult male voxel phantom constructed on the basis of CT images," Radiat. Prot. Dosim., 123[3], 337 (2007).

11) G. Tanaka, H. Kawamura, Anatomical and physiological characteristics for Asian reference man -Male and female of different ages-, NIRS-M-115, National Institute on Radiological Sciences (NIRS) (1996).

12) K. V. Riper, D. L. Spikes, R. G. McDowell, "Variable human anthropomorphic models," Proc. 1996 Radiation Protection and Shielding Division Topical Meeting (American Nuclear Society), Cape Cod, USA, Apr.21, 1996, 643 (1996).

13) Y. Yamaguchi, "JEUNESS: A computer code to calculate photon external doses using age-specific phantoms," Hoken-butsuri (J. Jpn. Health Phys.), 27[4], 305 (1992), [in Japanese].

14) H. Iwase, K. Niita, T. Nakamura, "Development of generalpurpose particle and heavy ion transport Monte Carlo code," J. Nucl. Sci. Technol., 39 [11], 1142 (2002).

15) D. G. Jones, P. C. Shrimpton, Survey of CT practice in UL -Part III Normalized organ doses calculated using Monte Carlo techniques-, NRPB-R250, National Radiological Protection Board (NRPB) (1991).

16) R. Nowotny, A.Hvfer, "Ein Programm fur die Berechnung von diagnostischen Roentgenspektren, “ Fortschr. Roentgenstr., 142, 685 (1985), [in German]. 